\title{
Ihr Wissen für kommende Generationen - im Hufeland-Vademecum
}

Liebe Ärztinnen und Ärzte,

täglich kommen Patientinnen und Patienten zu Ihnen. Sie vertrauen darauf, dass Sie aus dem vielfältigen Schatz an Arzneimitteln der Komplementärmedizin das richtige Mittel auswählen und verordnen. Dabei greifen Sie auf Ihr vielfältiges Erfahrungswissen zurück, dass Sie in den verschiedenen Ausbildungen, in Fortbildungen, durch Kollegen und durch Ihre eigene jahrelange Erfahrung in der praktischen Anwendung erworben haben.

Stellen Sie sich vor, Sie würden heute anfangen, auf diesem Gebiet zu praktizieren. Wie gut wäre da ein Nachschlagewerk, das in einfacher und systematischer Weise das Wissen und die Erfahrungen zu den verschiedenen Arzneimitteln bündelt. Stellen Sie sich vor, welcher Wissensschatz der nachfolgenden Generation zur Verfügung gestellt werden könnte, wenn Sie und Ihre Kolle- gen ihre Erfahrungen für die Zukunft festhalten. Arbeiten Sie mit am Hufeland-Vademecum.

\section{Erfahrungswissen teilen}

Die Hufelandgesellschaft ruft deshalb die komplementärmedizinisch tätigen Ärztinnen und Ärzte dazu auf, ihr Erfahrungswissen zu teilen und in der online zugänglichen Datenbank einzugeben. Mittels des Fragebogens werden systematisch unterschiedliche Aspekte zur Verordnung von der Indikation und Dosierung bis hin zur Wirkung erfasst.

Alle Datenbankeinträge werden gesammelt, gesichtet und auf Vollständigkeit geprüft. Auch kritische Berichte werden aufgenommen und dokumentiert. Ihre erfahrungsbasierten Eingaben werden ergänzt durch Literaturnachweise und Evidenzen.

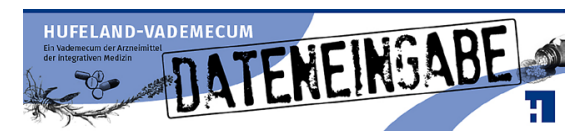

\section{Qualitätssicherung}

Glaubwürdigkeit und Gültigkeit der Einträge sind für die Qualitätssicherung des Vademecums von herausragender Bedeutung. Deshalb wird eine Bewertungskommission von Expertinnen und Experten mit ausgezeichneter und praktischer Fachkenntnis zur Anwendung von komplementärmedizinischen Arzneimitteln die Erfahrungsberichte sichten und über die Freigabe und Veröffentlichung entscheiden.

Mehr zum Projekt und den Zugang zur Datenbank finden Sie auf www.hufelandgesellschaft.de. 